\title{
The split equilibrium problem and its convergence algorithms
}

Zhenhua $\mathrm{He}^{*}$

"Correspondence: zhenhuahe@126.com

Department of Mathematics, Honghe University, Mengzi, Yunnan 661100, China

\begin{abstract}
The purpose of this paper is to introduce a split equilibrium problem (SEP) and find a solution of the equilibrium problem such that its image under a given bounded linear operator is a solution of another equilibrium problem. By using the iterative method, we construct some iterative algorithms to solve such problem in real Hilbert spaces and obtain some strong and weak convergence theorems. Finally, we point out that there exist many SEPs which need the use of new methods to solve them. Some examples are given to illustrate our results.
\end{abstract}

MSC: 47J25; 47H09; 65K10

Keywords: iterative algorithm; split equilibrium problem; strong and weak convergence

\section{Introduction}

Throughout this paper, the symbols $\mathbb{N}$ and $\mathbb{R}$ are used to denote the sets of positive integers and real numbers, respectively.

In this paper, we propose a new equilibrium problem, which is called a split equilibrium problem (SEP). Let $E_{1}$ and $E_{2}$ be two real Banach spaces. Let $C$ be a closed convex subset of $E_{1}, K$ a closed convex subset of $E_{2}$, and $A: E_{1} \rightarrow E_{2}$ a bounded linear operator. $f$ is a bi-function from $C \times C$ into $\mathbb{R}$ and $g$ is a bi-function from $K \times K$ into $\mathbb{R}$. The SEP is

to find an element $p \in C \quad$ such that $f(p, y) \geq 0, \quad \forall y \in C$,

and

such that $u:=A p \in K \quad$ solves $\quad g(u, v) \geq 0, \quad \forall v \in K$.

If we consider only the problem (1.1), then (1.1) is a classical equilibrium problem. From (1.1) and (1.2), we can see that the SEP contains two equilibrium problems, and the image of a solution of one equilibrium problem under a given bounded linear operator is a solution of another equilibrium problem. Since many problems coming from physics, optimization, and economics reduce to find a solution of the equilibrium problem (1.1) (see, for instance, $[1,2])$, the equilibrium problem (1.1) is very important in the field of applied mathematics. Some authors have proposed some methods to find the solution of the equilibrium problem (1.1). As a generalization of the equilibrium problem (1.1), when finding a

(c) 2012 He; licensee Springer. This is an Open Access article distributed under the terms of the Creative Commons Attribution License (http://creativecommons.org/licenses/by/2.0), which permits unrestricted use, distribution, and reproduction in any medium, provided the original work is properly cited. 
common solution for some equilibrium problems, it has been considered in the same subset of the same space; see [3-5]. However, in general, some equilibrium problems always belong to different subsets of spaces, so the SEP is important and quite general. The SEP should enable us to split the solution between two different subsets of spaces so that the image of a solution point of one problem, under a given bounded linear operator, is a solution point of another problem. A special case of the SEP is the split variational inequality problem (SVIP); see [6].

For convenience, in this paper let $\mathrm{EP}(f), \mathrm{EP}(g)$ and $\Omega=\{p \in \mathrm{EP}(f): A p \in \mathrm{EP}(g)\}$ denote the solution set of (1.1), (1.2) and the SEP, respectively.

Example 1.1 Let $E_{1}=E_{2}=\mathbb{R}, C:=[1,+\infty)$ and $K:=(-\infty,-4]$. Let $A(x)=-4 x$ for all $x \in \mathbb{R}$, then $A$ is a bounded linear operator. Let $f: C \times C \rightarrow \mathbb{R}$, and $g: K \times K \rightarrow \mathbb{R}$ be defined by $f(x, y)=y-x, g(u, v)=2(u-v)$, respectively. Clearly, $\operatorname{EP}(f)=\{1\}$ and $A(1)=-4 \in \operatorname{EP}(g)$. So $\Omega=\{p \in \mathrm{EP}(f): A p \in \mathrm{EP}(g)\} \neq \emptyset$.

Example 1.2 Let $E_{2}=\mathbb{R}$ with the standard norm $|\cdot|$ and $E_{1}=\mathbb{R}^{2}$ with the norm $\|\alpha\|=$ $\left(a_{1}^{2}+a_{2}^{2}\right)^{\frac{1}{2}}$ for some $\alpha=\left(a_{1}, a_{2}\right) \in \mathbb{R}^{2} . K:=[1,+\infty)$ and $C:=\left\{\alpha=\left(a_{1}, a_{2}\right) \in \mathbb{R}^{2} \mid a_{2}-a_{1} \geq 1\right\}$. Define a bi-function $f(w, \alpha)=w_{1}-w_{2}+a_{2}-a_{1}$, where $w=\left(w_{1}, w_{2}\right), \alpha=\left(a_{1}, a_{2}\right) \in C$, then $f$ is a bi-function from $C \times C$ into $\mathbb{R}$ with $\operatorname{EP}(f)=\left\{p=\left(p_{1}, p_{2}\right) \mid p_{2}-p_{1}=1\right\}$. For each $\alpha=$ $\left(a_{1}, a_{2}\right) \in E_{1}$, let $A \alpha=a_{2}-a_{1}$, then $A$ is a bounded linear operator from $E_{1}$ into $E_{2}$. In fact, it is also easy to verify that $A\left(a \alpha_{1}+b \alpha_{2}\right)=a A\left(\alpha_{1}\right)+b A\left(\alpha_{2}\right)$ and $\|A\|=\sqrt{2}$ for some $\alpha_{1}, \alpha_{2} \in E_{1}$ and $a, b \in \mathbb{R}$. Now define another bi-function $g$ as follows: $g(u, v)=v-u$ for all $u, v \in K$. Then $g$ is a bi-function from $K \times K$ into $\mathbb{R}$ with $\operatorname{EP}(g)=\{1\}$.

Clearly, when $p \in \operatorname{EP}(f)$, we have $A p=1 \in \mathrm{EP}(g)$. So $\Omega=\{p \in \operatorname{EP}(f): A p \in \operatorname{EP}(g)\} \neq \emptyset$.

Remark 1.1 The SEP in Example 1.1 lies in two different subsets of the same space. While the SEP in Example 1.2 lies in two different subsets of the different space.

In this paper, we construct some iterative algorithms to solve the SEP. Some strong and weak convergence theorems are established. The results obtained in this paper can be reckoned as the new development of the equilibrium problem (1.1). Finally, we point out that there exist many SEPs which need the use of new methods to solve them. Some examples are given to illustrate our results.

\section{Preliminaries}

We assume that $H$ is a real Hilbert space with zero vector $\theta$ whose inner product and norm are denoted by $\langle\cdot, \cdot\rangle$ and $\|\cdot\|$, respectively; and we use symbols $\rightarrow$ and $\rightarrow$ to denote strong and weak convergence, respectively.

Let $H_{1}$ and $H_{2}$ be two Hilbert spaces. The operator $A$ from $H_{1}$ into $H_{2}$ and the operator $B$ from $H_{2}$ into $H_{1}$ are two bounded linear operators. $B$ is called the adjoint operator of $A$, if for all $z \in H_{1}, w \in H_{2}, B$ satisfies $\langle A z, w\rangle=\langle z, B w\rangle$. Especially, if $H_{1}=H_{2}$, then $B$ reduces to the well-known adjoint operator of $A$.

Remark 2.1 It is easy to verify that the operator $B$, an adjoint operator of $A$, has the following characters:

(i) $\|B\|=\|A\|$; (ii) $B$ is a unique adjoint operator of $A$. 
Example 2.1 Let $H_{2}=\mathbb{R}$ with the standard norm $|\cdot|$ and $H_{1}=\mathbb{R}^{2}$ with the norm $\|\alpha\|=$ $\left(a_{1}^{2}+a_{2}^{2}\right)^{\frac{1}{2}}$ for some $\alpha=\left(a_{1}, a_{2}\right) \in \mathbb{R}^{2} .\langle x, y\rangle=x y$ denotes the inner product of $H_{2}$ for some $x, y \in H_{2}$ and $\langle\alpha, \beta\rangle=a_{1} b_{1}+a_{2} b_{2}$ denotes the inner product of $H_{1}$ for some $\alpha=\left(a_{1}, a_{2}\right)$, $\beta=\left(b_{1}, b_{2}\right) \in H_{1}$. Let $A \alpha=a_{2}-a_{1}$, then $A$ is a bounded linear operator from $H_{1}$ into $H_{2}$ with $\|A\|=\sqrt{2}$. For $x \in H_{2}$, let $B x=(-x, x)$, then $B$ is a bounded linear operator from $H_{2}$ into $H_{1}$ with $\|B\|=\sqrt{2}$. Moreover, for any $\alpha=\left(a_{1}, a_{2}\right) \in H_{1}$ and $x \in H_{2},\langle A \alpha, x\rangle=\langle\alpha, B x\rangle$, so $B$ is an adjoint operator of $A$.

Example 2.2 Let $H_{1}=\mathbb{R}^{2}$ with the norm $\|\alpha\|=\left(a_{1}^{2}+a_{2}^{2}\right)^{\frac{1}{2}}$ for some $\alpha=\left(a_{1}, a_{2}\right) \in \mathbb{R}^{2}$ and $H_{2}=\mathbb{R}^{3}$ with the norm $\|\gamma\|=\left(c_{1}^{2}+c_{2}^{2}+c_{3}^{2}\right)^{\frac{1}{2}}$ for some $\gamma=\left(c_{1}, c_{2}, c_{3}\right) \in \mathbb{R}^{3}$. Let $\langle\alpha, \beta\rangle=a_{1} b_{1}+a_{2} b_{2}$ and $\langle\gamma, \eta\rangle=c_{1} d_{1}+c_{2} d_{2}+c_{3} d_{3}$ denote the inner product of $H_{1}$ and $H_{2}$, respectively, where $\alpha=\left(a_{1}, a_{2}\right), \beta=\left(b_{1}, b_{2}\right) \in H_{1}, \gamma=\left(c_{1}, c_{2}, c_{3}\right), \eta=\left(d_{1}, d_{2}, d_{3}\right) \in \mathbb{R}^{3}$. Let $A \alpha=\left(a_{2}, a_{1}, a_{1}-a_{2}\right)$ for $\alpha=\left(a_{1}, a_{2}\right) \in H_{1}$, then $A$ is a bounded linear operator from $H_{1}$ into $H_{2}$ with $\|A\|=\sqrt{3}$ because $\left\|\left(\frac{\sqrt{2}}{2},-\frac{\sqrt{2}}{2},-\sqrt{2}\right)\right\| \leq \sup _{\|\alpha\|=1}\|A \alpha\| \leq \sqrt{3}$. For $\gamma=\left(c_{1}, c_{2}, c_{3}\right) \in$ $H_{2}$, let $B \gamma=\left(c_{2}+c_{3}, c_{1}-c_{3}\right)$, then $B$ is a bounded linear operator from $H_{2}$ into $H_{1}$ with $\|B\|=\sqrt{3}$. Moreover, for any $\alpha=\left(a_{1}, a_{2}\right) \in H_{1}$ and $\gamma=\left(c_{1}, c_{2}, c_{3}\right) \in H_{2},\langle A \alpha, \gamma\rangle=\langle\alpha, B \gamma\rangle$, so $B$ is an adjoint operator of $A$.

Let $K$ be a closed convex subset of a real Hilbert space $H$. For each point $x \in H$, there exists a unique nearest point in $K$, denoted by $P_{K} x$, such that

$$
\left\|x-P_{K} x\right\| \leq\|x-y\|, \quad \forall y \in K .
$$

The mapping $P_{K}$ is called the metric projection from $H$ onto $K$. It is well known that $P_{K}$ has the following characters:

(i) $\left\langle x-y, P_{K} x-P_{K} y\right\rangle \geq\left\|P_{K} x-P_{K} y\right\|^{2}$ for every $x, y \in H$.

(ii) For $x \in H$, and $z \in K, z=P_{K}(x) \Leftrightarrow\langle x-z, z-y\rangle \geq 0, \forall y \in K$.

(iii) For $x \in H$ and $y \in K$,

$$
\left\|y-P_{K}(x)\right\|^{2}+\left\|x-P_{K}(x)\right\|^{2} \leq\|x-y\|^{2} .
$$

A Banach space $(X,\|\cdot\|)$ is said to satisfy Opial's condition if, for each sequence $\left\{x_{n}\right\}$ in $X$ which converges weakly to a point $x \in X$, we have

$$
\liminf _{n \rightarrow \infty}\left\|x_{n}-x\right\|<\liminf _{n \rightarrow \infty}\left\|x_{n}-y\right\|, \quad \forall y \in X, y \neq x .
$$

It is well known that each Hilbert space satisfies Opial's condition.

The following results are crucial to our main results.

Lemma 2.1 (see [1]) Let $K$ be a nonempty closed convex subset of $H$ and $F$ be a bi-function of $K \times K$ into $\mathbb{R}$ satisfying the following conditions:

(A1) $F(x, x)=0$ for all $x \in K$;

(A2) $F$ is monotone, that is, $F(x, y)+F(y, x) \leq 0$ for all $x, y \in K$;

(A3) for each $x, y, z \in K$,

$$
\limsup _{t \downarrow 0} F(t z+(1-t) x, y) \leq F(x, y)
$$


(A4) for each $x \in K, y \mapsto F(x, y)$ is convex and lower semi-continuous.

Let $r>0$ and $x \in H$. Then, there exists $z \in K$ such that

$$
F(z, y)+\frac{1}{r}\langle y-z, z-x\rangle \geq 0, \quad \text { for all } y \in K
$$

Lemma 2.2 (see [7]) Let $K$ be a nonempty closed convex subset of $H$ and let $F$ be a bifunction of $K \times K$ into $\mathbb{R}$ satisfying (A1)-(A4). For $r>0$ and $x \in H$, define a mapping $T_{r}^{F}$ : $H \rightarrow K$ as follows:

$$
T_{r}^{F}(x)=\left\{z \in K: F(z, y)+\frac{1}{r}\langle y-z, z-x\rangle \geq 0, \forall y \in K\right\}
$$

for all $x \in H$. Then the following hold:

(i) $T_{r}^{F}$ is single-valued;

(ii) $T_{r}^{F}$ is firmly non-expansive, that is, for any $x, y \in H$,

$$
\left\|T_{r}^{F} x-T_{r}^{F} y\right\|^{2} \leq\left\langle T_{r}^{F} x-T_{r}^{F} y, x-y\right\rangle
$$

(iii) $F\left(T_{r}^{F}\right)=\mathrm{EP}(F)$ for $\forall r>0$;

(iv) $\mathrm{EP}(F)$ is closed and convex.

Lemma 2.3 (see [3]) Let $H$ be a real Hilbert space. Then for any $x_{1}, x_{2}, \ldots, x_{k} \in H$ and $a_{1}, a_{2}, \ldots, a_{k} \in[0,1]$ with $\sum_{i=1}^{k} a_{i}=1, k \in \mathbb{N}$, we have

$$
\left\|\sum_{i=1}^{k} a_{i} x_{i}\right\|^{2}=\sum_{i=1}^{k} a_{i}\left\|x_{i}\right\|^{2}-\sum_{i=1}^{k-1} \sum_{j=i+1}^{k} a_{i} a_{j}\left\|x_{i}-x_{j}\right\|^{2} .
$$

In particular, we have

(1) $\|\alpha x+(1-\alpha) y\|^{2}=\alpha\|x\|^{2}+(1-\alpha)\|y\|^{2}-\alpha(1-\alpha)\|x-y\|^{2}$ for all $x, y \in H$ and $\alpha \in[0,1]$

(2) the map $f: H \rightarrow \mathbb{R}$ defined by $f(x)=\|x\|^{2}$ is convex.

Lemma 2.4 (see, e.g., [8]) Let $H$ be a real Hilbert space. Then the following hold:

(a) $\|x+y\|^{2} \leq\|y\|^{2}+2\langle x, x+y\rangle$ for all $x, y \in H$

(b) $\|x-y\|^{2}=\|x\|^{2}+\|y\|^{2}-2\langle x, y\rangle$ for all $x, y \in H$.

Lemma 2.5 Let $K$ be a nonempty closed convex subset of $H$. For $x \in H$, let the mapping $T_{r}^{F}(x)$ be the same as in Lemma 2.2. Then for $r, s>0$ and $x, y \in H$,

$$
\left\|T_{r}^{F}(x)-T_{s}^{F}(y)\right\| \leq\|y-x\|+\frac{|s-r|}{s}\left\|T_{s}^{F}(y)-y\right\| .
$$

Proof For $r, s>0$ and $x, y \in H$, by (i) of Lemma 2.2, we can let $z_{1}=T_{r}^{F}(x)$ and $z_{2}=T_{s}^{F}(y)$. By the definition of $T_{r}^{F}$, we have

$$
F\left(z_{1}, u\right)+\frac{1}{r}\left\langle u-z_{1}, z_{1}-x\right\rangle \geq 0, \quad \forall u \in K
$$


and

$$
F\left(z_{2}, u\right)+\frac{1}{s}\left\langle u-z_{2}, z_{2}-y\right\rangle \geq 0, \quad \forall u \in K
$$

Taking $u=z_{2}$ in (2.3) and $u=z_{1}$ in (2.4), we have

$$
F\left(z_{1}, z_{2}\right)+\frac{1}{r}\left\langle z_{2}-z_{1}, z_{1}-x\right\rangle \geq 0
$$

and

$$
F\left(z_{2}, z_{1}\right)+\frac{1}{s}\left\langle z_{1}-z_{2}, z_{2}-y\right\rangle \geq 0
$$

Since the bi-function $F$ satisfies the condition (A2), from (2.5) and (2.6) we have

$$
\frac{1}{r}\left\langle z_{2}-z_{1}, z_{1}-x\right\rangle+\frac{1}{s}\left\langle z_{1}-z_{2}, z_{2}-y\right\rangle \geq 0,
$$

which implies that

$$
\left\langle z_{2}-z_{1}, z_{1}-x\right\rangle-\left\langle z_{2}-z_{1}, r \frac{z_{2}-y}{s}\right\rangle \geq 0
$$

Thus, we have

$$
\left\langle z_{2}-z_{1}, z_{1}-z_{2}+z_{2}-x-\frac{r}{s}\left(z_{2}-y\right)\right\rangle \geq 0
$$

this implies that

$$
\left\|z_{2}-z_{1}\right\|^{2} \leq\left\langle z_{2}-z_{1}, z_{2}-x-\frac{r}{s}\left(z_{2}-y\right)\right\rangle \leq\left\|z_{2}-z_{1}\right\|\left\|z_{2}-x-\frac{r}{s}\left(z_{2}-y\right)\right\|
$$

SO

$$
\begin{aligned}
\left\|z_{2}-z_{1}\right\| & \leq\left\|z_{2}-x-\frac{r}{s}\left(z_{2}-y\right)\right\| \leq\|y-x\|+\left\|\left(1-\frac{r}{s}\right)\left(z_{2}-y\right)\right\| \\
& =\|y-x\|+\frac{|s-r|}{s}\left\|T_{s}^{F}(y)-y\right\|,
\end{aligned}
$$

namely,

$$
\left\|T_{r}^{F}(x)-T_{s}^{F}(y)\right\| \leq\|y-x\|+\frac{|s-r|}{s}\left\|T_{s}^{F}(y)-y\right\| .
$$

The proof is completed.

\section{Main results}

In this section, we will solve the SEP which satisfies the conditions (A1)-(A4).

Theorem 3.1 (Weak convergence theorem) Let $C$ be a nonempty closed convex subset of $H_{1}$ and $K$ a nonempty closed convex subset of $H_{2}$, where $H_{1}$ and $H_{2}$ are two real Hilbert 
spaces. $\wedge:=\{1,2, \ldots, k\}$ denotes a finite index set. For any $i \in \wedge, f_{i}: C \times C \rightarrow \mathbb{R}$ is a bifunction with $\bigcap_{i=1}^{k} \operatorname{EP}\left(f_{i}\right) \neq \emptyset$. Let $A: H_{1} \rightarrow H_{2}$ be a bounded linear operator with the adjoint $B$ and $g: K \times K \rightarrow \mathbb{R}$ a bi-function with $\operatorname{EP}(g) \neq \emptyset$. Let $\left\{x_{n}\right\}$ and $\left\{u_{n}^{i}\right\}(i \in \wedge)$ be sequences generated by

$$
\left\{\begin{array}{l}
x_{1} \in C, \\
f_{i}\left(u_{n}^{i}, y\right)+\frac{1}{r_{n}}\left\langle y-u_{n}^{i}, u_{n}^{i}-x_{n}\right\rangle \geq 0, \quad y \in C, i \in \wedge, \\
\tau_{n}=\frac{u_{n}^{1}+\cdots+u_{n}^{k}}{k}, \\
g\left(w_{n}, z\right)+\frac{1}{r_{n}}\left\langle z-w_{n}, w_{n}-A \tau_{n}\right\rangle \geq 0, \quad z \in K, \\
x_{n+1}=P_{C}\left(\tau_{n}+\mu B\left(w_{n}-A \tau_{n}\right)\right), \quad \forall n \in \mathbb{N},
\end{array}\right.
$$

where $\left\{r_{n}\right\} \subset(0,+\infty)$ with $\liminf _{n \rightarrow \infty} r_{n}>0, P_{C}$ is a projection operator from $H_{1}$ into $C$ and $\mu \in\left(0, \frac{1}{\|B\|^{2}}\right)$ is a constant. Suppose that $\Omega=\left\{p \in \bigcap_{i=1}^{k} \operatorname{EP}\left(f_{i}\right): A p \in \operatorname{EP}(g)\right\} \neq \emptyset$, then the sequences $\left\{x_{n}\right\}$ and $\left\{u_{n}^{i}\right\}(i \in \wedge)$ converge weakly to an element $p \in \Gamma$, while $\left\{w_{n}\right\}$ converges weakly to $A p \in \mathrm{EP}(g)$.

Proof For each $i \in \wedge$ and each $r>0$, let $T_{r}^{f_{i}}: H_{1} \rightarrow C$ be defined by (2.2), then $u_{n}^{i}=T_{r_{n}}^{f_{i}} x_{n}$ for all $n \in \mathbb{N}$ by Lemma 2.2. Again let $T_{r}^{g}$ : $H_{2} \rightarrow K$ be defined by (2.2), then $w_{n}=T_{r_{n}}^{g} A \tau_{n}$ for all $n \in \mathbb{N}$. So (3.1) can be rewritten as follows:

$$
\left\{\begin{array}{l}
x_{1} \in C, \\
u_{n}^{i}=T_{r_{n}}^{f_{i}} x_{n}, \quad i \in \wedge, \\
\tau_{n}=\frac{u_{n}^{1}+\cdots+u_{n}^{k}}{k}, \\
w_{n}=T_{r_{n}}^{g} A \tau_{n}, \\
x_{n+1}=P_{C}\left(\tau_{n}+\mu B\left(T_{r_{n}}^{g}-I\right) A \tau_{n}\right), \quad \forall n \in \mathbb{N} .
\end{array}\right.
$$

Let $x^{*} \in C$ be a point such that $x^{*} \in \bigcap_{i=1}^{k} \mathrm{EP}\left(f_{i}\right)$ and $A x^{*} \in \mathrm{EP}(g)$, namely, $x^{*} \in \Omega$. By Lemma 2.2 and Lemma 2.4, it follows that

$$
\begin{aligned}
\left\|u_{n}^{i}-x^{*}\right\|^{2} & =\left\|T_{r_{n}}^{f_{i}} x_{n}-T_{r_{n}}^{f_{i}} x^{*}\right\|^{2} \leq\left\langle T_{r_{n}}^{f_{i}} x_{n}-T_{r_{n}}^{f_{i}} x^{*}, x_{n}-x^{*}\right\rangle \\
& =\frac{1}{2}\left\{\left\|u_{n}^{i}-x^{*}\right\|^{2}+\left\|x_{n}-x^{*}\right\|^{2}-\left\|u_{n}^{i}-x_{n}\right\|^{2}\right\},
\end{aligned}
$$

hence

$$
\left\|u_{n}^{i}-x^{*}\right\|^{2} \leq\left\|x_{n}-x^{*}\right\|^{2}-\left\|u_{n}^{i}-x_{n}\right\|^{2} .
$$

Applying Lemma 2.3, we get

$$
\left\|\tau_{n}-x^{*}\right\|^{2} \leq \frac{1}{k} \sum_{i=1}^{k}\left\|u_{n}^{i}-x^{*}\right\|^{2} .
$$


(3.3) and (3.4) imply that

$$
\left\|\tau_{n}-x^{*}\right\|^{2} \leq\left\|x_{n}-x^{*}\right\|^{2}-\frac{1}{k} \sum_{i=1}^{k}\left\|u_{n}^{i}-x_{n}\right\|^{2} \text {. }
$$

Again from Lemma 2.2, we have

$$
\left\|w_{n}-A x^{*}\right\|=\left\|T_{r_{n}}^{g} A \tau_{n}-A x^{*}\right\| \leq\left\|A \tau_{n}-A x^{*}\right\| \quad \text { for each } n \in \mathbb{N} \text {. }
$$

By (b) of Lemma 2.4 and (3.6), for each $n \in \mathbb{N}$, we have

$$
\begin{aligned}
2 \mu & \left\langle\tau_{n}-x^{*}, B\left(T_{r_{n}}^{g}-I\right) A \tau_{n}\right\rangle \\
= & 2 \mu\left\langle A\left(\tau_{n}-x^{*}\right)+\left(T_{r_{n}}^{g}-I\right) A \tau_{n}-\left(T_{r_{n}}^{g}-I\right) A \tau_{n},\left(T_{r_{n}}^{g}-I\right) A \tau_{n}\right\rangle \\
= & 2 \mu\left(\left\langle T_{r_{n}}^{g} A \tau_{n}-A x^{*},\left(T_{r_{n}}^{g}-I\right) A \tau_{n}\right\rangle-\left\|\left(T_{r_{n}}^{g}-I\right) A \tau_{n}\right\|^{2}\right) \\
= & 2 \mu\left(\frac{1}{2}\left\|T_{r_{n}}^{g} A \tau_{n}-A x^{*}\right\|^{2}+\frac{1}{2}\left\|\left(T_{r_{n}}^{g}-I\right) A \tau_{n}\right\|^{2}\right. \\
& \left.-\frac{1}{2}\left\|A \tau_{n}-A x^{*}\right\|^{2}-\left\|\left(T_{r_{n}}^{g}-I\right) A \tau_{n}\right\|^{2}\right) \\
\leq & 2 \mu\left(\frac{1}{2}\left\|\left(T_{r_{n}}^{g}-I\right) A \tau_{n}\right\|^{2}-\left\|\left(T_{r_{n}}^{g}-I\right) A \tau_{n}\right\|^{2}\right) \\
= & -\mu\left\|\left(T_{r_{n}}^{g}-I\right) A \tau_{n}\right\|^{2} .
\end{aligned}
$$

We also have

$$
\left\|B\left(T_{r_{n}}^{g}-I\right) A \tau_{n}\right\|^{2} \leq\|B\|^{2}\left\|\left(T_{r_{n}}^{g}-I\right) A \tau_{n}\right\|^{2}
$$

From (3.2), (3.5)-(3.8), we have

$$
\begin{aligned}
\left\|x_{n+1}-x^{*}\right\|^{2} & =\left\|P_{C}\left(\tau_{n}+\mu B\left(T_{r_{n}}^{g}-I\right) A \tau_{n}\right)-P_{C} x^{*}\right\|^{2} \\
& \left.\leq \| \tau_{n}+\mu B\left(T_{r_{n}}^{g}-I\right) A \tau_{n}\right)-x^{*} \|^{2} \\
& =\left\|\tau_{n}-x^{*}\right\|^{2}+\left\|\mu B\left(T_{r_{n}}^{g}-I\right) A \tau_{n}\right\|^{2}+2 \mu\left\langle\tau_{n}-x^{*}, B\left(T_{r_{n}}^{g}-I\right) A \tau_{n}\right\rangle \\
& \leq\left\|\tau_{n}-x^{*}\right\|^{2}+\mu^{2}\|B\|^{2}\left\|\left(T_{r_{n}}^{g}-I\right) A \tau_{n}\right\|^{2}-\mu\left\|\left(T_{r_{n}}^{g}-I\right) A \tau_{n}\right\|^{2} \\
& =\left\|\tau_{n}-x^{*}\right\|^{2}-\mu\left(1-\mu\|B\|^{2}\right)\left\|\left(T_{r_{n}}^{g}-I\right) A \tau_{n}\right\|^{2} \\
& \leq\left\|x_{n}-x^{*}\right\|^{2}-\mu\left(1-\mu\|B\|^{2}\right)\left\|\left(T_{r_{n}}^{g}-I\right) A \tau_{n}\right\|^{2} .
\end{aligned}
$$

Notice $\mu \in\left(0, \frac{1}{\|B\|^{2}}\right), \mu\left(1-\mu\|B\|^{2}\right)>0$. It follows from (3.9) that

$$
\left\|x_{n+1}-x^{*}\right\| \leq\left\|\tau_{n}-x^{*}\right\| \leq\left\|x_{n}-x^{*}\right\|
$$

and

$$
\mu\left(1-\mu\|B\|^{2}\right)\left\|\left(T_{r_{n}}^{g}-I\right) A \tau_{n}\right\|^{2} \leq\left\|x_{n}-x^{*}\right\|^{2}-\left\|x_{n+1}-x^{*}\right\|^{2} .
$$


(3.10) implies $\lim _{n \rightarrow \infty}\left\|x_{n}-x^{*}\right\|$ exists. Further, from (3.10)-(3.11),

$$
\lim _{n \rightarrow \infty}\left\|x_{n}-x^{*}\right\|=\lim _{n \rightarrow \infty}\left\|\tau_{n}-x^{*}\right\|, \quad \lim _{n \rightarrow \infty}\left\|\left(T_{r_{n}}^{g}-I\right) A \tau_{n}\right\|=0
$$

Again from (3.5), we have

$$
\lim _{n \rightarrow \infty}\left\|u_{n}^{i}-x_{n}\right\|=0, \quad i \in \wedge
$$

which yields that

$$
\lim _{n \rightarrow \infty}\left\|\left(T_{r_{n}}^{f_{i}}-I\right) x_{n}\right\|=\lim _{n \rightarrow \infty}\left\|T_{r_{n}}^{f_{i}} x_{n}-x_{n}\right\|=\lim _{n \rightarrow \infty}\left\|u_{n}^{i}-x_{n}\right\|=0, \quad i \in \wedge,
$$

and

$$
\left\|\tau_{n}-x_{n}\right\| \leq\left\|u_{n}^{1}-x_{n}\right\|+\cdots+\left\|u_{n}^{k}-x_{n}\right\| \rightarrow 0 \quad \text { as } n \rightarrow \infty .
$$

Because $\lim _{n \rightarrow \infty}\left\|x_{n}-x^{*}\right\|$ exists, which implies $\left\{x_{n}\right\}$ is bounded, hence $\left\{x_{n}\right\}$ has a weakly convergence subsequence $\left\{x_{n_{j}}\right\}$. Assume that $x_{n_{j}} \rightarrow p$ for some $p \in C$. Then $u_{n_{j}}^{i} \rightarrow p, \tau_{n_{j}} \rightarrow$ $p$ and $A \tau_{n_{j}} \rightarrow A p \in K$ by (3.13) and (3.15).

Now we prove $p \in \Omega$ or, to be more precise, we prove $p \in \bigcap_{i=1}^{k} \operatorname{EP}\left(f_{i}\right)$ and $A p \in \operatorname{EP}(g)$. By Lemma 2.2, for any $r>0, \operatorname{EP}\left(f_{i}\right)=F\left(T_{r}^{f_{i}}\right), i \in \wedge$, and $\operatorname{EP}(g)=F\left(T_{r}^{g}\right)$. For $i \in \wedge$, since $\left(I-T_{r_{n}}^{f_{i}}\right) x_{n} \rightarrow 0$ by (3.14), we have $T_{r}^{f_{i}} p=p$ for $r>0$. Otherwise, if $T_{r}^{f_{i}} p \neq p$ for all $i \in \wedge$, then by Opial's condition, we have

$$
\begin{aligned}
\liminf _{j \rightarrow \infty}\left\|x_{n_{j}}-p\right\| & <\liminf _{j \rightarrow \infty}\left\|x_{n_{j}}-T_{r}^{f_{i}} p\right\| \\
& =\liminf _{j \rightarrow \infty}\left\|x_{n_{j}}-T_{r_{n_{j}}}^{f_{i}} x_{n_{j}}+T_{r_{n_{j}}}^{f_{i}} x_{n_{j}}-T_{r}^{f_{i}} p\right\| \\
& \leq \liminf _{j \rightarrow \infty}\left\{\left\|x_{n_{j}}-T_{r_{n_{j}}}^{f_{i}} x_{n_{j}}\right\|+\left\|T_{r_{n_{j}}}^{f_{i}} x_{n_{j}}-T_{r}^{f_{i}} p\right\|\right\} \\
& =\liminf _{j \rightarrow \infty}\left\|T_{r_{n_{j}}}^{f_{i}} x_{n_{j}}-T_{r}^{f_{i}} p\right\| \\
& =\liminf _{j \rightarrow \infty}\left\|T_{r}^{f_{i}} p-T_{r_{n_{j}}}^{f_{i}} x_{n_{j}}\right\| \\
& \leq \liminf _{j \rightarrow \infty}\left(\left\|x_{n_{j}}-p\right\|+\frac{\left|r_{n_{j}}-r\right|}{r_{n_{j}}}\left\|T_{r_{n_{j}}}^{f_{i}} x_{n_{j}}-x_{n_{j}}\right\|\right) \\
& =\liminf _{j \rightarrow \infty}\left\|x_{n_{j}}-p\right\|,
\end{aligned}
$$

this is a contradiction. So this shows that $p \in \bigcap_{i=1}^{k} F\left(T_{r}^{f_{i}}\right)=\bigcap_{i=1}^{k} \operatorname{EP}\left(f_{i}\right)$. Similarly, we can prove $A p \in \mathrm{EP}(g)$.

Finally, we prove $\left\{x_{n}\right\}$ and $\left\{u_{n}^{i}\right\}$ converge weakly to $p \in \Omega$, while $\left\{w_{n}\right\}$ converges weakly to $A p \in \mathrm{EP}(g)$. Firstly, if there exists other subsequence of $\left\{x_{n}\right\}$ which is denoted by $\left\{x_{n_{t}}\right\}$ such that $x_{n_{t}} \rightarrow q \in \Omega$ with $q \neq p$, then, by Opial's condition,

$$
\liminf _{t \rightarrow \infty}\left\|x_{n_{t}}-q\right\|<\liminf _{t \rightarrow \infty}\left\|x_{n_{t}}-p\right\|<\liminf _{t \rightarrow \infty}\left\|x_{n_{t}}-q\right\| .
$$

This is a contradiction. Hence $\left\{x_{n}\right\}$ and $\left\{u_{n}^{i}\right\}$ converge weakly to $p \in \Omega$, respectively. 
On the other hand, by (3.15) we also have $\tau_{n} \rightarrow p$. Notice that $\left\|w_{n}-A \tau_{n}\right\|=\|\left(T_{r_{n}}^{g}-\right.$ I) $A \tau_{n} \| \rightarrow 0$ by (3.12), so we have $\tau_{n} \rightarrow A p$ and $w_{n} \rightarrow A p$. We obtain the desired result.

Corollary 3.1 Let $C$ be a nonempty closed convex subset of $H_{1}$ and $K$ a nonempty closed convex subset of $H_{2}$, where $H_{1}$ and $H_{2}$ are two real Hilbert spaces. $f: C \times C \rightarrow \mathbb{R}$ is a bifunction with $\mathrm{EP}(f) \neq \emptyset$. Let $A: H_{1} \rightarrow H_{2}$ be a bounded linear operator with the adjoint $B$ and $g: K \times K \rightarrow \mathbb{R}$ a bi-function with $\mathrm{EP}(g) \neq \emptyset$. Let $\left\{x_{n}\right\}$ and $\left\{u_{n}\right\}$ be sequences generated by

$$
\left\{\begin{array}{l}
x_{1} \in C, \\
f\left(u_{n}, y\right)+\frac{1}{r_{n}}\left\langle y-u_{n}, u_{n}-x_{n}\right\rangle \geq 0, \quad y \in C, \\
g\left(w_{n}, z\right)+\frac{1}{r_{n}}\left\langle z-w_{n}, w_{n}-A u_{n}\right\rangle \geq 0, \quad z \in K, \\
x_{n+1}=P_{C}\left(u_{n}+\mu B\left(w_{n}-A u_{n}\right)\right), \quad \forall n \in \mathbb{N},
\end{array}\right.
$$

where $\left\{r_{n}\right\} \subset(0,+\infty)$ with $\liminf _{n \rightarrow \infty} r_{n}>0, P_{C}$ is a projection operator from $H_{1}$ into $C$ and $\mu \in\left(0, \frac{1}{\|B\|^{2}}\right)$ is a constant. Suppose that $\Omega=\{p \in \mathrm{EP}(f): A p \in \operatorname{EP}(g)\} \neq \emptyset$, then the sequences $\left\{x_{n}\right\}$ and $\left\{u_{n}\right\}$ converge weakly to an element $p \in \Omega$, while $\left\{w_{n}\right\}$ converges weakly to $A p \in \mathrm{EP}(g)$.

Theorem 3.2 (Strong convergence theorem) Let $C$ be a nonempty closed convex subset of $H_{1}$ and $K$ a nonempty closed convex subset of $H_{2}$, where $H_{1}$ and $H_{2}$ are two real Hilbert spaces. $\wedge:=\{1,2, \ldots, k\}$ denotes a finite index set. For any $i \in \wedge, f_{i}: C \times C \rightarrow \mathbb{R}$ is a bifunction with $\bigcap_{i=1}^{k} \operatorname{EP}\left(f_{i}\right) \neq \emptyset$. Let $A: H_{1} \rightarrow H_{2}$ be a bounded linear operator with the adjoint $B$ and $g: K \times K \rightarrow \mathbb{R}$ a bi-function with $\mathrm{EP}(g) \neq \emptyset$. Let $C_{1}=C$ and $\left\{x_{n}\right\}$ and $\left\{u_{n}^{i}\right\}$ $(i \in \wedge)$ be sequences generated by

$$
\left\{\begin{array}{l}
x_{1} \in C, \\
f_{i}\left(u_{n}^{i}, y\right)+\frac{1}{r_{n}}\left\langle y-u_{n}^{i}, u_{n}^{i}-x_{n}\right\rangle \geq 0, \quad y \in C, i \in \wedge, \\
\tau_{n}=\frac{u_{n}^{1}+\cdots+u_{n}^{k}}{k}, \\
g\left(w_{n}, z\right)+\frac{1}{r_{n}}\left\langle z-w_{n}, w_{n}-A \tau_{n}\right\rangle \geq 0, \quad z \in K, \\
y_{n}=P_{C}\left(\tau_{n}+\mu B\left(w_{n}-A \tau_{n}\right)\right), \\
C_{n+1}=\left\{v \in C_{n}:\left\|y_{n}-v\right\| \leq\left\|\tau_{n}-v\right\| \leq\left\|x_{n}-v\right\|\right\}, \\
x_{n+1}=P_{C_{n+1}}\left(x_{1}\right), \quad n \in \mathbb{N},
\end{array}\right.
$$

where $\left\{r_{n}\right\} \subset(0,+\infty)$ with $\liminf _{n \rightarrow \infty} r_{n}>0, P_{C}$ is a projection operator from $H_{1}$ into $C$ and $\mu \in\left(0, \frac{1}{\|B\|^{2}}\right)$ is a constant. Suppose that $\Omega=\left\{p \in \bigcap_{i=1}^{k} \operatorname{EP}\left(f_{i}\right): A p \in \operatorname{EP}(g)\right\} \neq \emptyset$, then the sequences $\left\{x_{n}\right\}$ and $\left\{u_{n}^{i}\right\}(i \in \wedge)$ converge strongly to an element $x^{\prime \prime} \in \Omega$, while $\left\{w_{n}\right\}$ converges strongly to $A x^{*} \in \mathrm{EP}(g)$.

Proof By Lemma 2.2, $u_{n}^{i}=T_{r_{n}}^{f_{i}} x_{n}$ for all $i \in \wedge, n \in \mathbb{N}$ and $w_{n}=T_{r_{n}}^{g} A \tau_{n}$ for all $n \in \mathbb{N}$. We claim $C_{n} \neq \emptyset$ for $n \in \mathbb{N}$. In fact $\Omega \subset C_{n}$ for $n \in \mathbb{N}$. Indeed, let $p \in \Omega$, it follows from (3.7) and (3.8) that

$$
2 \mu\left\langle\tau_{n}-p, B\left(T_{r_{n}}^{g}-I\right) A \tau_{n}\right\rangle \leq-\mu\left\|\left(T_{r_{n}}^{g}-I\right) A \tau_{n}\right\|^{2},
$$


and

$$
\left\|B\left(T_{r_{n}}^{g}-I\right) A \tau_{n}\right\|^{2} \leq\|B\|^{2}\left\|\left(T_{r_{n}}^{g}-I\right) A \tau_{n}\right\|^{2}
$$

From (3.17)-(3.19) we have

$$
\begin{aligned}
\left\|y_{n}-p\right\|^{2} & \leq\left\|\tau_{n}+\mu B\left(T_{r_{n}}^{g}-I\right) A \tau_{n}-p\right\|^{2} \\
& =\left\|\tau_{n}-p\right\|^{2}+\left\|\mu B\left(T_{r_{n}}^{g}-I\right) A \tau_{n}\right\|^{2}+2 \mu\left\langle\tau_{n}-p, B\left(T_{r_{n}}^{g}-I\right) A \tau_{n}\right\rangle \\
& \leq\left\|\tau_{n}-p\right\|^{2}+\mu^{2}\|B\|^{2}\left\|\left(T_{r_{n}}^{g}-I\right) A \tau_{n}\right\|^{2}-\mu\left\|\left(T_{r_{n}}^{g}-I\right) A \tau_{n}\right\|^{2} \\
& =\left\|\tau_{n}-p\right\|^{2}-\mu\left(1-\mu\|B\|^{2}\right)\left\|\left(T_{r_{n}}^{g}-I\right) A \tau_{n}\right\|^{2} \\
& \leq\left\|x_{n}-p\right\|^{2}-\mu\left(1-\mu\|B\|^{2}\right)\left\|\left(T_{r_{n}}^{g}-I\right) A \tau_{n}\right\|^{2} .
\end{aligned}
$$

Notice $\mu \in\left(0, \frac{1}{\|B\|^{2}}\right), \mu\left(1-\mu\|B\|^{2}\right)>0$. It follows from (3.20) that

$$
\left\|y_{n}-p\right\| \leq\left\|\tau_{n}-p\right\| \leq\left\|x_{n}-p\right\| \quad \text { for all } n \in \mathbb{N}
$$

this shows $p \in C_{n}$ for all $n \in \mathbb{N}$, so $\Omega \subset C_{n}$ and $C_{n} \neq \emptyset$ for $n \in \mathbb{N}$.

We want to prove $C_{n}$ is a closed convex set for $n \in \mathbb{N}$. It is easy to verify that $C_{n}$ is closed for $n \in \mathbb{N}$, so it suffices to verify $C_{n}$ is convex for $n \in \mathbb{N}$. In fact, let $v_{1}, v_{2} \in C_{n+1}$, for each $\lambda \in(0,1)$, we have

$$
\begin{aligned}
\left\|y_{n}-\left(\lambda v_{1}+(1-\lambda) \nu_{2}\right)\right\|^{2} & =\left\|\lambda\left(y_{n}-v_{1}\right)+(1-\lambda)\left(y_{n}-v_{2}\right)\right\|^{2} \\
& =\lambda\left\|y_{n}-v_{1}\right\|^{2}+(1-\lambda)\left\|y_{n}-v_{2}\right\|^{2}-\lambda(1-\lambda)\left\|v_{1}-v_{2}\right\|^{2} \\
& \leq \lambda\left\|\tau_{n}-v_{1}\right\|^{2}+(1-\lambda)\left\|\tau_{n}-v_{2}\right\|^{2}-\lambda(1-\lambda)\left\|v_{1}-v_{2}\right\|^{2} \\
& =\left\|\tau_{n}-\left(\lambda v_{1}+(1-\lambda) v_{2}\right)\right\|^{2},
\end{aligned}
$$

namely, $\left\|y_{n}-\left(\lambda v_{1}+(1-\lambda) v_{2}\right)\right\| \leq\left\|\tau_{n}-\left(\lambda v_{1}+(1-\lambda) v_{2}\right)\right\|$. Similarly, we have $\| \tau_{n}-\left(\lambda v_{1}+\right.$ $\left.(1-\lambda) v_{2}\right)\|\leq\| x_{n}-\left(\lambda v_{1}+(1-\lambda) v_{2}\right) \|$, this shows $\lambda v_{1}+(1-\lambda) v_{2} \in C_{n+1}$ and $C_{n+1}$ is a convex set for $n \in \mathbb{N}$.

By (iv) of Lemma 2.2, $\Omega$ is a closed convex set, so there exists a unique element $q=$ $P_{\Omega}\left(x_{1}\right) \in \Omega \subset C_{n}$. Since $x_{n}=P_{C_{n}}\left(x_{1}\right)$, we have $\left\|x_{n}-x_{1}\right\| \leq\left\|q-x_{1}\right\|$, which shows that $\left\{x_{n}\right\}$ is bounded. So are $\left\{\tau_{n}\right\}$ and $\left\{y_{n}\right\}$. Notice that $C_{n+1} \subset C_{n}$ and $x_{n+1}=P_{C_{n+1}}\left(x_{1}\right) \subset C_{n}$, then

$$
\left\|x_{n+1}-x_{1}\right\| \leq\left\|x_{n}-x_{1}\right\|, \quad n \geq 2
$$

It follows that $\lim _{n \rightarrow \infty}\left\|x_{n}-x_{0}\right\|$ exists.

For some $m, n \in \mathbb{N}$ with $m>n$, from $x_{m}=P_{C_{m}}\left(x_{1}\right) \subset C_{n}$ and (2.1), we have

$$
\left\|x_{n}-x_{m}\right\|^{2}+\left\|x_{1}-x_{m}\right\|^{2}=\left\|x_{n}-P_{C_{m}}\left(x_{1}\right)\right\|^{2}+\left\|x_{1}-P_{C_{m}}\left(x_{0}\right)\right\|^{2} \leq\left\|x_{n}-x_{1}\right\|^{2} .
$$

By (3.22)-(3.23) we have $\lim _{n \rightarrow \infty}\left\|x_{n}-x_{m}\right\|=0$, so $\left\{x_{n}\right\}$ is a Cauchy sequence. Let $x_{n} \rightarrow x^{*}$. 
Next we prove $x^{*} \in \Omega$. Firstly, by $x_{n+1}=P_{C_{n+1}}\left(x_{1}\right) \in C_{n+1} \subset C_{n}$, from (3.17) we have

$$
\begin{aligned}
\left\|y_{n}-x_{n}\right\| & \leq\left\|y_{n}-x_{n+1}\right\|+\left\|x_{n+1}-x_{n}\right\| \leq 2\left\|x_{n+1}-x_{n}\right\| \rightarrow 0, \\
\left\|\tau_{n}-x_{n}\right\| & \leq\left\|\tau_{n}-x_{n+1}\right\|+\left\|x_{n+1}-x_{n}\right\| \leq 2\left\|x_{n+1}-x_{n}\right\| \rightarrow 0, \\
\left\|y_{n}-\tau_{n}\right\| & \leq\left\|y_{n}-x_{n}\right\|+\left\|x_{n}-\tau_{n}\right\| \rightarrow 0 .
\end{aligned}
$$

Again from (3.20), we have

$$
\begin{aligned}
\left\|\left(T_{r_{n}}^{g}-I\right) A \tau_{n}\right\|^{2} & \leq \frac{1}{\mu\left(1-\mu\|B\|^{2}\right)}\left\{\left\|x_{n}-p\right\|^{2}-\left\|y_{n}-p\right\|^{2}\right\} \\
& \leq \frac{1}{\mu\left(1-\mu\|B\|^{2}\right)}\left\|x_{n}-y_{n}\right\|\left\{\left\|x_{n}-p\right\|+\left\|y_{n}-p\right\|\right\} \rightarrow 0 .
\end{aligned}
$$

So

$$
\lim _{n \rightarrow \infty}\left\|\left(T_{r_{n}}^{g}-I\right) A \tau_{n}\right\|=0
$$

Notice $\tau_{n}=\frac{u_{n}^{1}+\cdots+u_{n}^{k}}{k}$, hence from (3.24) and (3.5), we obtain

$$
\lim _{n \rightarrow \infty}\left\|T_{r_{n}}^{f_{i}} x_{n}-x_{n}\right\|=\lim _{n \rightarrow \infty}\left\|u_{n}^{i}-x_{n}\right\|=0, \quad i \in \wedge
$$

Since $x_{n} \rightarrow x^{*},(3.27)$ and Lemma 2.5 imply that for $r>0$,

$$
\begin{aligned}
\left\|T_{r}^{f_{i}} x^{*}-x^{*}\right\| & \leq\left\|T_{r}^{f_{i}} x^{*}-T_{r_{n}}^{f_{i}} x_{n}\right\|+\left\|T_{r_{n}}^{f_{i}} x_{n}-x_{n}\right\|+\left\|x_{n}-x^{*}\right\| \\
& \leq\left\|x_{n}-x^{*}\right\|+\frac{\left|r_{n}-r\right|}{r_{n}}\left\|T_{r_{n}}^{f_{i}} x_{n}-x_{n}\right\|+\left\|T_{r_{n}}^{f_{i}} x_{n}-x_{n}\right\|+\left\|x_{n}-x^{*}\right\| \rightarrow 0,
\end{aligned}
$$

which yields that $x^{*} \in F\left(T_{r}^{f_{i}}\right)$ for all $i \in \wedge$, further $x^{*} \in \bigcap_{i=1}^{k} \operatorname{EP}\left(f_{i}\right)$. Since $A$ is a bounded linear operator, $\left\|A x_{n}-A x^{*}\right\| \rightarrow 0$ by $x_{n} \rightarrow x^{*}$. Then for $r>0$, by (3.26) and Lemma 2.5 we have

$$
\begin{aligned}
\left\|T_{r}^{g} A x^{*}-A x^{*}\right\| \leq & \left\|T_{r}^{g} A x^{*}-T_{r_{n}}^{g} A x_{n}\right\|+\left\|T_{r_{n}}^{g} A x_{n}-A x_{n}\right\|+\left\|A x_{n}-A x^{*}\right\| \\
\leq & \left\|A x_{n}-A x^{*}\right\|+\frac{\left|r_{n}-r\right|}{r_{n}}\left\|T_{r_{n}}^{g} A x_{n}-A x_{n}\right\|+\left\|T_{r_{n}}^{g} A x_{n}-A x_{n}\right\| \\
& +\left\|A x_{n}-A x^{*}\right\| \rightarrow 0
\end{aligned}
$$

hence, $A x^{*} \in F\left(T_{r}^{g}\right)=\mathrm{EP}(g)$ for $r>0$. Thus we have proved $x^{*} \in \Omega$, namely, $\left\{x_{n}\right\}$ converges strongly to $x^{*} \in \Omega$. Notice (3.27), we also have $\left\{u_{n}^{i}\right\}(i \in \wedge)$ converges strongly to $x^{*} \in \Omega$.

Since $\left\|\tau_{n}-x_{n}\right\| \rightarrow 0$ by (3.24), we have $\tau_{n} \rightarrow x^{*}$ by $x_{n} \rightarrow x^{*}$. Again from (3.26) we have

$$
\lim _{n \rightarrow \infty}\left\|w_{n}-A \tau_{n}\right\|=\lim _{n \rightarrow \infty}\left\|\left(T_{r_{n}}^{g}-I\right) A \tau_{n}\right\|=0
$$

hence $w_{n} \rightarrow A x^{*} \in \mathrm{EP}(g)$. The proof is completed. 
Corollary 3.2 Let $C$ be a nonempty closed convex subset of $H_{1}$ and $K$ a nonempty closed convex subset of $H_{2}$, where $H_{1}$ and $H_{2}$ are two real Hilbert spaces. $f: C \times C \rightarrow \mathbb{R}$ is a bifunction with $\mathrm{EP}(f) \neq \emptyset$. Let $A: H_{1} \rightarrow H_{2}$ be a bounded linear operator with the adjoint $B$ and $g: K \times K \rightarrow \mathbb{R}$ a bi-function with $\mathrm{EP}(g) \neq \emptyset$. Let $C_{1}=C$ and $\left\{x_{n}\right\}$ and $\left\{u_{n}\right\}$ be sequences generated by

$$
\left\{\begin{array}{l}
x_{1} \in C, \\
f\left(u_{n}, y\right)+\frac{1}{r_{n}}\left\langle y-u_{n}, u_{n}-x_{n}\right\rangle \geq 0, \quad y \in C, \\
g\left(w_{n}, z\right)+\frac{1}{r_{n}}\left\langle z-w_{n}, w_{n}-A u_{n}\right\rangle \geq 0, \quad z \in K, \\
y_{n}=P_{C}\left(u_{n}+\mu B\left(w_{n}-A u_{n}\right)\right), \\
C_{n+1}=\left\{v \in C_{n}:\left\|y_{n}-v\right\| \leq\left\|u_{n}-v\right\| \leq\left\|x_{n}-v\right\|\right\}, \\
x_{n+1}=P_{C_{n+1}}\left(x_{1}\right), \quad n \in \mathbb{N},
\end{array}\right.
$$

where $\left\{r_{n}\right\} \subset(0,+\infty)$ with $\liminf _{n \rightarrow \infty} r_{n}>0, \mu \in\left(0, \frac{1}{\|B\|^{2}}\right)$ is a constant. Suppose that $\Omega=$ $\{p \in \operatorname{EP}(f): A p \in \operatorname{EP}(g)\} \neq \emptyset$. Then the sequences $\left\{x_{n}\right\}$ and $\left\{u_{n}\right\}$ converge strongly to an element $x^{*} \in \Omega$, while $\left\{w_{n}\right\}$ converges strongly to $A x^{*} \in \mathrm{EP}(g)$.

If $C=H_{1}$ and $K=H_{2}$ in Theorem 3.1 and Theorem 3.2, we have the following corollaries.

Corollary 3.3 Let $H_{1}$ and $H_{2}$ be two real Hilbert spaces. $\wedge:=\{1,2, \ldots, k\}$ denotes a finite index set. For any $i \in \wedge, f_{i}: H_{1} \times H_{1} \rightarrow \mathbb{R}$ is a bi-function with $\bigcap_{i=1}^{k} \mathrm{EP}\left(f_{i}\right) \neq \emptyset$. Let $A: H_{1} \rightarrow$ $\mathrm{H}_{2}$ be a bounded linear operator with the adjoint $B$ and $g: H_{2} \times H_{2} \rightarrow \mathbb{R}$ a bi-function with $\mathrm{EP}(g) \neq \emptyset$. Let $\left\{x_{n}\right\}$ and $\left\{u_{n}^{i}\right\}$ be sequences generated by

$$
\left\{\begin{array}{l}
x_{1} \in H_{1}, \\
f_{i}\left(u_{n}^{i}, y\right)+\frac{1}{r_{n}}\left\langle y-u_{n}^{i}, u_{n}^{i}-x_{n}\right\rangle \geq 0, y \in H_{1}, \quad i \in \wedge, \\
\tau_{n}=\frac{u_{n}^{1}+\cdots+u_{n}^{k}}{k}, \\
g\left(w_{n}, z\right)+\frac{1}{r_{n}}\left\langle z-w_{n}, w_{n}-A \tau_{n}\right\rangle \geq 0, \quad z \in H_{2}, \\
x_{n+1}=\tau_{n}+\mu B\left(w_{n}-A \tau_{n}\right), \quad \forall n \in \mathbb{N},
\end{array}\right.
$$

where $\left\{r_{n}\right\} \subset(0,+\infty)$ with $\liminf _{n \rightarrow \infty} r_{n}>0, \mu \in\left(0, \frac{1}{\|B\|^{2}}\right)$ is a constant. Suppose that $\Omega=$ $\left\{p \in \bigcap_{i=1}^{k} \operatorname{EP}\left(f_{i}\right): A p \in \operatorname{EP}(g)\right\} \neq \emptyset$. Then the sequences $\left\{x_{n}\right\}$ and $\left\{u_{n}^{i}\right\}(i \in \wedge)$ converge weakly to an element $p \in \Omega$, while $\left\{w_{n}\right\}$ converges weakly to $A p \in \mathrm{EP}(g)$.

Corollary 3.4 Let $H_{1}$ and $H_{2}$ be two real Hilbert spaces. $f: H_{1} \times H_{1} \rightarrow \mathbb{R}$ is a bi-function with $\mathrm{EP}(f) \neq \emptyset$. Let $A: H_{1} \rightarrow H_{2}$ be a bounded linear operator with the adjoint $B$ and $g:$ $H_{2} \times H_{2} \rightarrow \mathbb{R}$ a bi-function with $\mathrm{EP}(g) \neq \emptyset$. Let $\left\{x_{n}\right\}$ and $\left\{u_{n}\right\}$ be sequences generated by

$$
\left\{\begin{array}{l}
x_{1} \in H_{1}, \\
f\left(u_{n}, y\right)+\frac{1}{r_{n}}\left\langle y-u_{n}, u_{n}-x_{n}\right\rangle \geq 0, \quad y \in H_{1}, \\
g\left(w_{n}, z\right)+\frac{1}{r_{n}}\left\langle z-w_{n}, w_{n}-A u_{n}\right\rangle \geq 0, \quad z \in H_{2}, \\
x_{n+1}=u_{n}+\mu B\left(w_{n}-A u_{n}\right), \quad \forall n \in \mathbb{N},
\end{array}\right.
$$


where $\left\{r_{n}\right\} \subset(0,+\infty)$ with $\liminf _{n \rightarrow \infty} r_{n}>0, \mu \in\left(0, \frac{1}{\|B\|^{2}}\right)$ is a constant. Suppose that $\Omega=\{p \in \mathrm{EP}(f): A p \in \mathrm{EP}(g)\} \neq \emptyset$. Then the sequences $\left\{x_{n}\right\}$ and $\left\{u_{n}\right\}$ converge weakly to an element $p \in \Omega$, while $\left\{w_{n}\right\}$ converges weakly to $A p \in \mathrm{EP}(g)$.

Corollary 3.5 Let $H_{1}$ and $H_{2}$ be two real Hilbert spaces. $\wedge:=\{1,2, \ldots, k\}$ denotes a finite index set. For any $i \in \wedge, f_{i}: H_{1} \times H_{1} \rightarrow \mathbb{R}$ is a bi-function with $\bigcap_{i=1}^{k} \mathrm{EP}\left(f_{i}\right) \neq \emptyset$. Let $A: H_{1} \rightarrow$ $\mathrm{H}_{2}$ be a bounded linear operator with the adjoint $B$ and $g: H_{2} \times H_{2} \rightarrow \mathbb{R}$ a bi-function with $\mathrm{EP}(g) \neq \emptyset$. Let $C_{1}=H_{1}$ and $\left\{x_{n}\right\}$ and $\left\{u_{n}^{i}\right\}(i \in \wedge)$ be sequences generated by

$$
\left\{\begin{array}{l}
x_{1} \in H_{1}, \\
f_{i}\left(u_{n}^{i}, y\right)+\frac{1}{r_{n}}\left\langle y-u_{n}^{i}, u_{n}^{i}-x_{n}\right\rangle \geq 0, \quad y \in H_{1}, i \in \wedge, \\
\tau_{n}=\frac{u_{n}^{1}+\cdots+u_{n}^{k}}{k}, \\
g\left(w_{n}, z\right)+\frac{1}{r_{n}}\left\langle z-w_{n}, w_{n}-A \tau_{n}\right\rangle \geq 0, \quad z \in H_{2}, \\
y_{n}=\tau_{n}+\mu B\left(w_{n}-A \tau_{n}\right), \\
C_{n+1}=\left\{v \in C_{n}:\left\|y_{n}-v\right\| \leq\left\|\tau_{n}-v\right\| \leq\left\|x_{n}-v\right\|\right\}, \\
x_{n+1}=P_{C_{n+1}}\left(x_{1}\right), \quad n \in \mathbb{N},
\end{array}\right.
$$

where $\left\{r_{n}\right\} \subset(0,+\infty)$ with $\liminf _{n \rightarrow \infty} r_{n}>0, \mu \in\left(0, \frac{1}{\|B\|^{2}}\right)$ is a constant. Suppose that $\Omega=\left\{p \in \bigcap_{i=1}^{k} \operatorname{EP}\left(f_{i}\right): A p \in \operatorname{EP}(g)\right\} \neq \emptyset$. Then the sequences $\left\{x_{n}\right\}$ and $\left\{u_{n}^{i}\right\}(i \in \wedge)$ converge strongly to an element $p \in \Omega$, while $\left\{w_{n}\right\}$ converges strongly to $A x^{*} \in \operatorname{EP}(g)$.

Corollary 3.6 Let $H_{1}$ and $H_{2}$ be two real Hilbert spaces. $f: H_{1} \times H_{1} \rightarrow \mathbb{R}$ is a bi-function with $\mathrm{EP}(f) \neq \emptyset$. Let $A: H_{1} \rightarrow H_{2}$ be a bounded linear operator with the adjoint $B$ and $g$ : $H_{2} \times H_{2} \rightarrow \mathbb{R}$ a bi-function with $\mathrm{EP}(g) \neq \emptyset$. Let $C_{1}=H_{1}$ and $\left\{x_{n}\right\}$ and $\left\{u_{n}\right\}$ be sequences generated by

$$
\left\{\begin{array}{l}
x_{1} \in H_{1}, \\
f\left(u_{n}, y\right)+\frac{1}{r_{n}}\left\langle y-u_{n}, u_{n}-x_{n}\right\rangle \geq 0, \quad y \in H_{1}, \\
g\left(w_{n}, z\right)+\frac{1}{r_{n}}\left\langle z-w_{n}, w_{n}-A u_{n}\right\rangle \geq 0, \quad z \in H_{2}, \\
y_{n}=u_{n}+\mu B\left(w_{n}-A u_{n}\right), \\
C_{n+1}=\left\{v \in C_{n}:\left\|y_{n}-v\right\| \leq\left\|u_{n}-v\right\| \leq\left\|x_{n}-v\right\|\right\}, \\
x_{n+1}=P_{C_{n+1}}\left(x_{1}\right), \quad n \in \mathbb{N},
\end{array}\right.
$$

where $\left\{r_{n}\right\} \subset(0,+\infty)$ with $\liminf _{n \rightarrow \infty} r_{n}>0, \mu \in\left(0, \frac{1}{\|B\|^{2}}\right)$ is a constant. Suppose that $\Omega=$ $\{p \in \operatorname{EP}(f): A p \in \operatorname{EP}(g)\} \neq \emptyset$. Then the sequences $\left\{x_{n}\right\}$ and $\left\{u_{n}\right\}$ converge strongly to an element $x^{*} \in \Omega$, while $\left\{w_{n}\right\}$ converges strongly to $A x^{\prime \prime} \in \operatorname{EP}(g)$.

Remark 3.1 Since Example 1.1 and Example 1.2 satisfy the conditions of Corollary 3.1 and Corollary 3.2, the SEPs in Example 1.1 and Example 1.2 can be solved by the algorithm (3.16) and (3.28). 
Remark 3.2 The results of this paper provide some solution algorithms for some SEPs; however, there are still some SEPs which cannot be solved by the results of this paper. The following examples belong to the case.

Example 3.1 Let $H_{2}=\mathbb{R}$ and $H_{1}=\mathbb{R}^{2}$ with the norm $\|z\|=\left(x^{2}+y^{2}\right)^{\frac{1}{2}}$ for some $z=(x, y) \in$ $\mathbb{R}^{2} . K:=[1,+\infty)$ and $C:=\left\{z=(x, y) \in \mathbb{R}^{2} \mid y-x \geq 1\right\}$. Define a bi-function $f(w, z)=x_{1}+y_{1}+$ $y_{2}-x_{2}$, where $w=\left(x_{1}, y_{1}\right), z=\left(x_{2}, y_{2}\right) \in C$, then $f$ is a bi-function from $C \times C$ into $\mathbb{R}$ with $\operatorname{EP}(f)=\{w=(x, y) \mid y-x \geq 1, x+y \geq-1\}$. For each $z=(x, y) \in H_{1}$, let $A z=y-x$, then $A$ is a bounded linear operator from $H_{1}$ into $H_{2}$. Now define another bi-function $g$ as follows: $g(u, v)=v-u$ for all $u, v \in K$. Then $g$ is a bi-function from $K \times K$ into $\mathbb{R}$ with $\operatorname{EP}(g)=\{1\}$.

Clearly, when $p=(x, y) \in \mathrm{EP}(f)$ with $y-x=1$ and $x+y \geq-1$, we have $A p=1 \in \mathrm{EP}(g)$. So $\Gamma=\{p \in \operatorname{EP}(f): A p \in \operatorname{EP}(g)\} \neq \emptyset$. However, because the bi-function $f$ does not satisfy the conditions (A1)-(A4), the SEP in this example cannot be solved by Corollary 3.1 or Corollary 3.2.

Example 3.2 Let $H_{1}, H_{2}, A$ and $B$ be the same as Example 2.2. Let $C:=\left\{\alpha=\left(a_{1}, a_{2}\right) \in\right.$ $\left.H_{1} \mid a_{2}-a_{1} \geq 1\right\}$ and $K:=\left\{\gamma=\left(c_{1}, c_{2}, c_{3}\right) \in H_{2} \mid\|\gamma\| \leq 2\right\}$. Define a bi-function $f(\alpha, \beta)=\left(b_{2}-\right.$ $\left.b_{1}\right)^{2}-\left(a_{1}+a_{2}\right)^{2}$, where $\alpha=\left(a_{1}, a_{2}\right), \beta=\left(b_{1}, b_{2}\right) \in C$, then $f$ is a bi-function from $C \times C$ into $\mathbb{R}$ with $\operatorname{EP}(f)=\left\{p=\left(p_{1}, p_{2}\right) \mid p_{2}-p_{1} \geq 1 \geq p_{1}+p_{2} \geq-1\right\}$. Define another bi-function $g(\gamma, \eta)=c_{2}^{2}+c_{3}^{2}-\left(c_{1}^{2}+d_{1}^{2}+d_{2}^{2}+d_{3}^{2}\right)$, where $\gamma=\left(c_{1}, c_{2}, c_{3}\right), \eta=\left(d_{1}, d_{2}, d_{3}\right) \in K$, then $\operatorname{EP}(g)=$ $\left\{u=\left(0, u_{2}, u_{3}\right) \in K \mid u_{2}^{2}+u_{3}^{2}=2\right\}$.

Clearly, when $p=(-1,0) \in \operatorname{EP}(f)$, we have $A p=(0,-1,-1) \in \operatorname{EP}(g)$. So $\Gamma=\{p \in \operatorname{EP}(f)$ : $A p \in \mathrm{EP}(g)\} \neq \emptyset$. However, since all $f$ and $g$ do not satisfy the conditions (A1)-(A4), we cannot use the results obtained in this paper to solve the SEP in this example.

\section{Conclusion}

There are still many SEPs which do not satisfy the conditions (A1)-(A4), so we need to develop some new methods to solve these problems in the future.

\section{Competing interests}

The author declares that he has no competing interests.

\section{Acknowledgement}

The author was supported by the Natural Science Foundation of Yunnan Province (2010ZC152).

Received: 1 April 2012 Accepted: 2 July 2012 Published: 20 July 2012

\section{References}

1. Blum, E, Oettli, W: From optimization and variational inequalities to equilibrium problems. Math. Stud. 63, 123-145 (1994)

2. Moudafi, A, Théra, M: Proximal and dynamical approaches to equilibrium problems. In: Lecture Notes in Economics and Mathematical Systems, vol. 477, pp. 187-201. Springer, Berlin (1999)

3. He, Z, Du, WS: Strong convergence theorems for equilibrium problems and fixed point problems: a new iterative method, some comments and applications. Fixed Point Theory Appl. 2011, 33 (2011)

4. Colao, V, Acedo, GL, Marino, G: An implicit method for finding common solutions of variational inequalities and systems of equilibrium problems and fixed points of infinite family of nonexpansive mappings. Nonlinear Anal. 71, 2708-2715 (2009)

5. Saeidi, S: Iterative algorithms for finding common solutions of variational inequalities and systems of equilibrium problems and fixed points of families and semigroups of nonexpansive mappings. Nonlinear Anal. 70, 4195-4208 (2009)

6. Censor, Y, Gibali, A, Reich, S: Algorithms for the split variational inequality problem. Numer. Algorithms 59(2), 301-323 (2012)

7. Combettes, PL, Hirstoaga, A: Equilibrium programming in Hilbert spaces. J. Nonlinear Convex Anal. 6, 117-136 (2005)

8. Chang, SS, Lee, HWJ, Chan, CK: A new method for solving equilibrium problem fixed point problem and variational inequality problem with application to optimization. Nonlinear Anal. 70, 3307-3319 (2009) 
doi:10.1186/1029-242X-2012-162

Cite this article as: He: The split equilibrium problem and its convergence algorithms. Journal of Inequalities and Applications 2012 2012:162.

Submit your manuscript to a SpringerOpen ${ }^{\circ}$ journal and benefit from:

- Convenient online submission

- Rigorous peer review

- Immediate publication on acceptance

- Open access: articles freely available online

- High visibility within the field

- Retaining the copyright to your article

Submit your next manuscript at $\gg$ springeropen.com 